\title{
Fructus Viticis methanolic extract attenuates trigeminal hyperalgesia in migraine by regulating injury signal transmission
}

\author{
WEN WEN ${ }^{1,2^{*}}$, HUAN CHEN $^{1,2^{*}}$, KUN FU $^{1,3^{*}}$, JIANGPING WEI $^{1,2}$, LIXIA QIN $^{1,2}$, TING PAN $^{1,2}$ and SHIJUN XU $^{1,2}$ \\ ${ }^{1}$ School of Pharmacy, Chengdu University of Traditional Chinese Medicine; ${ }^{2}$ Institute of Materia Medica Integration and \\ Transformation for Brain Disorders, Chengdu University of Traditional Chinese Medicine, Chengdu, Sichuan 611137; \\ ${ }^{3}$ Department of Pharmacy, The Third People's Hospital of Chengdu, The Second Affiliated Chengdu Clinical \\ College of Chongqing Medical University, Chengdu, Sichuan 610031, P.R. China
}

Received March 12, 2019; Accepted October 7, 2019

DOI: $10.3892 /$ etm.2019.8201

\begin{abstract}
Migraine, characterized by hyperalgesia of the trigeminovascular system, is a severe condition that leads to severe reductions in the quality of life. Upon external stimulation, the levels of various neurotransmitters, including aspartic acid (Asp), glutamic acid (Glu), $\gamma$-amino butyric acid (GABA), norepinephrine (NE) and 5-hydroxytryptamine (5-HT), are significantly altered; this directly or indirectly promotes trigeminal hypersensitivity. Fructus Viticis is a Traditional Chinese Medicine with analgesic properties to provide efficient relief of migraine. In the present study, the underlying mechanisms of the analgesic effect of Fructus Viticis methanolic extract were assessed in rats with nitroglycerin-induced migraine. The plasma levels of the neurotransmitters calcitonin gene-related peptide (CGRP) and substance P (SP), as well as the amount of c-fos immunoreactive cells (c-fos IR cells) in the brain, were detected. The analgesic effect was obvious, as Fructus Viticis methanolic extract ameliorated migraine-like behaviours in nitroglycerin-induced rats. The levels of 5-HT, GABA and NE in the brain of migraine model rats was lower compared with that of control rats, whereas opposite observations were made in the contents of excitatory amino acids. Pre-treatment with Fructus Viticis methanolic extract elevated the levels of 5-HT, GABA and NE, and also lowered the levels of excitatory amino acids, including Glu and Asp. In addition, treatment with Fructus Viticis methanolic extract lowered the plasma levels of CGRP and SP and decreased the c-fos IR cells in the brainstem. The present study provided a further scientific basis for the anti-migraine effects of Fructus Viticis.
\end{abstract}

Correspondence to: Professor Shijun Xu, School of Pharmacy, Chengdu University of Traditional Chinese Medicine, 1166 Liutai Street, Chengdu, Sichuan 611137, P.R. China

E-mail: xushijun@cdutcm.edu.cn

*Contributed equally

Key words: migraine, neurotransmitter, rats, Fructus Viticis

\section{Introduction}

Migraine is a neurological condition with common, chronic and multifactorial effects, characterized by severe attacks of headache and dysfunction in the autonomic nervous system. It is listed as one of the most debilitating neurological diseases and the seventh-highest cause of disability worldwide (1). Migraine affects $11 \%$ of adults, and is mostly associated with the 25-30 years age group (2). During migraine attacks, severe headache may last from hours to days and severely impairs the lives of affected individuals. In addition, migraine increases the risk of suffering from stroke or myocardial infarction (3).

The prevailing theory on the pathogenesis of migraine is that it arises from the trigeminovascular system (TMVS) (4). When stimulating the trigeminal nerve of a patient with migraine with either smell or pain, the blood oxygen level-dependent (BOLD) response increases in those patients. The BOLD response is an indirect method that measures neuronal activity by recording associated changes in cerebral hemodynamics (5). This functional response is associated with an increased patient pain rating on trigeminal stimulation. During a migraine headache, the TMVS becomes sensitized and excessively active for an extended period of time. The TMVS then releases excitatory neurotransmitters and pro-inflammatory mediators, including calcitonin gene-related peptide (CGRP), substance P (SP), 5-hydroxytryptamine (5-HT) and glutamic acid (Glu) (6). SP is a neurotransmitter that transmits pain, which produces potent vasodilatation via the direct (endothelium-independent) relaxation of vascular smooth muscle and has a potent role in relaxing blood vessels (7). These pro-inflammatory mediators may directly activate the nociceptors, generate action potentials or facilitate the firing of the nociceptors, resulting in peripheral sensitization and hyperalgesia $(8,9)$. They are also able to evoke the release of additional pro-inflammatory mediators, causing aseptic neuroinflammation (10). This may initiate the c-fos early gene expression and c-fos protein expression in the medulla oblongata, located in the brainstem (11). Changes in these neurotransmitters lead to strengthening of injury signals and eventually to migraine (12). Nitroglycerin is able to rapidly expand cerebral vessels and activate the trigeminal nucleus. The trigeminal nucleus then releases endogenous nitric oxide, keeping the TMVS sensitive (10). Therefore, the 
model generated using nitroglycerin to induce migraine in experimental animals is commonly used to study the effectiveness of novel drugs for treating migraine and the associated mechanisms (13).

According to previous studies, there are certain issues with drugs used to treat migraine, including drug resistance and dependence (14). Among triptan prescription medications, the most common side effects are racing heartbeat, warm sensations and chest pressure. Among other prescription medications, tiredness (69\%) and difficulty thinking clearly (35\%) were the most common side effects reported (15).

Traditional Chinese Medicine has the scope and long-reaching experience for the treatment of migraine $(16,17)$, with acupuncture being a good example.Scutellariabaicalensis, Du Liang soft capsule, and other Chinese patent medicines have also proved effective for migraine (18-21). Fructus Viticis is the dried fruit of Vitex trifolia L. var. simplicifolia cham. or Vitex trifolia L. The major active components of Fructus Viticis are flavonoids, diterpenes, steroids and iridoids (22). For quality control of Fructus Viticis, three flavonoids, 3,6,7-trimethylquercetagetin, casticin and luteolin, are used as reference substances (23). Fructus Viticis is widely used as a Traditional Chinese Medicine for treating migraine and its efficacy has been proven (24). However, it is currently unknown whether Fructus Viticis provides analgesia or inhibits neuroinflammation induced in the TMVS during migraine, and the potential roles of Fructus Viticis to inhibit pain signal transmission have remained elusive. Therefore, the present study was performed to investigate the mechanisms of action of Fructus Viticis to relieve migraine, and for this purpose, a migraine treatment experiment was designed and two classic analgesic experiments were used. The underlying mechanisms of the effects of Fructus Viticis methanolic extract (VME) in rats with nitroglycerin-induced migraine were explored by determining the plasma levels of Glu, aspartic acid (Asp), $\gamma$-aminobutyric acid (GABA), norepinephrine (NE), 5-HT, SP and CGRP, as well as c-fos-expressing cells in the brain stem.

\section{Materials and methods}

Chemical materials. Rizatriptan benzoate tablets were purchased from OuLi Pharmaceutical Co., Ltd.Fructus Viticis samples, which is the dry fruit of Vitex trifolia L, were obtained from the Hehuachi tradition herbal medicine market (Chengdu, China) and authenticated by Professor Yuntong Ma, Chengdu university of Traditional Chinese Medicine (Chengdu, China). The sample of the plant material was deposited into a Specimen bank of the Institute of Materia Medica Integration and Transformation for Brain Disorders (No. 510106180514WWen001). VME and rizatriptan benzoate tablets were respectively suspended in a $0.5 \%$ carboxymethyl cellulose (CMC)-Na solution for gastrointestinal administration. Nitroglycerin $(5 \mathrm{mg} / \mathrm{ml})$ was obtained from YiMin Pharmaceutical Co., Ltd. 5-HT, GABA, NE, Asp and Glu standards were purchased from Sigma-Aldrich (Merck KGaA). Rabbit polyclonal c-fos antibody (cat. no. ab209794) was provided by Abcam. Horseradish peroxidase-conjugated goat anti-rabbit IgG (cat. no. sc-2004) was obtained from Santa Cruz Biotechnology, Inc. Aspirin enteric-coated tablets were purchased from Bayer Healthcare Co., Ltd. Morphine hydrochloride injection was obtained from Shenyang First Pharmaceutical Co., Ltd. Normal goat serum was purchased from Zhejiang Tianhang Biotechnology Co., LTD. Acetic acid was purchased from Chengdu Kelong Chemical Reagent Factory. Chengdu Alfa Biotechnology Co., Ltd. provided casticin, luteolin and 3,6,7-trimethylquercetagetin. A SP ELISA kit (cat. no. ERC112-96) was purchased from ExCell Bio Biotechnology, Inc. A CGRP ELISA kit (cat. no. E-EL-R0135c) was obtained from Elabscience Biotechnology, Inc.

Preparation and analysis of VME extract. The whole Fructus Viticis samples were shredded and soaked for $30 \mathrm{~min}$ at room temperature each in volumes of methanol that were five times the weight of Fructus Viticis and underwent reflux extraction at $65^{\circ} \mathrm{C}$ for $30 \mathrm{~min}$. The extract was then collected, following which the aforementioned reflux extraction process was repeated again. For administration of the extract to the animals, the methanol was removed from the $V M E$ extract in advance in a vacuum using rotary evaporator where the residue was dissolved in $0.5 \% \mathrm{CMC}-\mathrm{Na}$ (used as the vehicle in the control and administration groups) at a concentration of $20 \mathrm{~g} / \mathrm{ml}$. The Agilent 1260 Infinity High-Performance Liquid Chromatography instrument (Agilent Technologies, Inc.) was used to analyze the extract solution and chromatographic separation was performed using an Ultimate AQ-C18 Column (4.6x250 mm, $5 \mu \mathrm{m}$; Agilent Technologies, Inc.) with a column temperature of $25^{\circ} \mathrm{C}$. A gradient-elution was provided with water as solvent $\mathrm{A}$ and acetonitrile as solvent $\mathrm{B}$, using a flow rate of $1.0 \mathrm{ml} / \mathrm{min}$, the elution started at 70:30 A:B (v/v) with the gradient gradually increased to 50:50 $(\mathrm{A}: \mathrm{B}, \mathrm{v} / \mathrm{v}$ ) for $15 \mathrm{~min}$, and maintained $15 \mathrm{~min}$, where $20 \mu \mathrm{l}$ sample was injected and the effluent absorbance was measured at $254 \mathrm{~nm}$. The concentration of luteolin, 3,6,7-trimethylquercetagetin and casticin was determined, which was performed using luteolin, 3,6,7-trimethylquercetagetin and casticin as reference compounds to verify the authenticity and quality of Fructus Viticis.

Animal experiments. All animal care and experimental procedures (including the analgesic experiments and migraine experiment) were approved by the Ethics Committee for Animal Experiments of the Institute of Materia Medica Integration and Transformation for Brain Disorders (Chengdu, China). A total of 60 male Sprague Dawley rats (weight, 250-300 g; age, 8 weeks) were purchased from the Experimental Animal Center of Sichuan Academy of Medical Sciences (certificate no. SCXK2013-15). Furthermore, 18 male Sprague Dawley rats (weight, 180-220 g; age, 6 weeks) were purchased from Chongqing Enswell Biotechnology Co., Ltd. (certificate no. SCXK20170003). A total of 48 female and 16 male Kunming mice (weight, 16-22 g; age, 5 weeks) were obtained from Dashuo Biological Technology Co., Ltd. (certificate no. SCXK2013-24). All of the animals were housed at a suitable temperature $\left(23 \pm 2^{\circ} \mathrm{C}\right)$ and humidity (40-70\%) with ad libitum access to water and food and a standard 12-h light/dark cycle, where the male animals were kept separately from the females. They were acclimatized to their housing environment for seven days prior to experimentation. All animal experiments complied with the Animal Research: Reporting in vivo Experiments guidelines and were performed 
in accordance with the National Institutes of Health (NIH) Guide for the Care and Use of Laboratory Animals (NIH Publication no. 8023, revised 1978).

\section{Analgesic experiments}

General. Two classic analgesic experiments were used to verify the analgesic effect of Fructus Viticis. One was the hot plate test and the other was the acetic acid-induced writhing test. Subsequently, a rat model was used to test the effect of Fructus Viticis on migraine. All products that were injected into the animals had been sterilized.

Hot plate test. The procedures performed in the present study was performed according to a previously published protocol (25). First, all female mice were placed on a hot plate at a temperature of $55 \pm 0.5^{\circ} \mathrm{C}$ to test the pain threshold; the pain reaction of the mice was paw licking (the time from placing on the hot plate to the occurrence of paw licking was recorded as the paw licking time). The mice were tested twice, with no less than 5 min between each test. Onset of paw licking was considered as the basic pain threshold. Mice with paw licking times of $>30 \mathrm{sec}$ and $<5 \mathrm{sec}$ were removed and the remaining female mice (with a paw licking time of 5-30 sec) were randomly divided into four groups. These included a control group, a morphine group, a VME high-dose group and a VME low-dose group ( $n=8$ for each group). The VME high-dose, VME low-dose, control and morphine groups were gastrointestinally administered with corresponding concentrations of VME or $0.5 \%$ CMC-Na. The oral gavage administration was given once a day for five days. At $30 \mathrm{~min}$ prior to the hot plate test, the morphine group received an intraperitoneal injection of $0.1 \%$ morphine hydrochloride $(0.01 \mathrm{ml} / \mathrm{g})$. The pain threshold of all groups was determined at 30,60,90 and 120 min after administration. If the paw licking time was $>60 \mathrm{sec}$, the pain threshold was recorded as $60 \mathrm{sec}$.

Acetic acid-induced writhing test. The procedures performed for this test were adopted from that reported previously (26). A total of 16 male and 16 female mice were randomly divided into four groups: A control group, aspirin group, VME high-dose group and VME extract low-dose group. The VME high-dose group, the VME low-dose group and the control group received gastrointestinal administration of corresponding concentrations of VME or $0.5 \% \mathrm{CMC}-\mathrm{Na}$. The aspirin group was administered with $0.6 \mathrm{~g} / \mathrm{kg}$ enteric-coated aspirin by oral gavage. The administration continued for 5 days, given once a day (27). Each mouse received $0.7 \%$ acetic acid solution in normal saline by intraperitoneal injection at a dosage of $0.2 \mathrm{ml}$ each hour after the last drug administration. The pain latency and number of times of writhing were observed and recorded within $15 \mathrm{~min}$.

Migraine experiment. The method used in the present study was according to a previously published protocol (21). A total of 60 rats were randomly divided into five groups: Control, model, rizatriptan, VME high-dose $(7 \mathrm{~g} / \mathrm{kg})$ and low-dose $(1.75 \mathrm{~g} / \mathrm{kg})$ groups $(\mathrm{n}=12)$. The dosage of Fructus Viticis for clinical use in human patients is $30 \mathrm{~g} /$ day, which may be converted to dose for rats of $3.5 \mathrm{~g} / \mathrm{kg} /$ day based on a weight change algorithm (28). The high-dose group received twice the clinical dose whereas the low-dose group received half the clinical dose (29). The clinical dose of rizatriptan for human patients is $10 \mathrm{mg} / \mathrm{d}$, which may be converted to $1 \mathrm{mg} / \mathrm{kg}$ for rats. The control group, model group, VME high-dose group and VME low-dose group received the corresponding concentrations of VME or $0.5 \%$ CMC-Na only by oral gavage administration. The administration continued for 15 days, given once a day.

At $30 \mathrm{~min}$ after the last administration, rats in the model, rizatriptan, VME high-dose and VME low-dose groups were injected subcutaneously with nitroglycerin at a dose of $10 \mathrm{mg} / \mathrm{kg}$. The migraine animal model used in the present study was based on that established in previous studies (30). The rats in the control group were injected subcutaneously with physiological saline at a dose of $2 \mathrm{ml} / \mathrm{kg}$. A few minutes later, the rats in the model group appeared restless, with characteristic symptoms of red ears, head scratching, cage climbing and body shaking. These symptoms lasted for $\sim 2 \mathrm{~h}$. Red ears, head scratching and cage climbing signs were more typical than other symptom characteristics. These three symptoms were therefore used to evaluate whether the migraine model was successfully established. For each rat, the latency of ear redness was recorded. If latency was $>60 \mathrm{~min}$, then the latency was recorded as $60 \mathrm{~min}$. The numbers of times of head scratching and cage climbing within 150 min were recorded.

Quantitative analysis of 5-HTe, NE, Glu, Asp and GABA. The method used was described previously (31-33). At $150 \mathrm{~min}$ after modeling, six randomly selected rats in each group were euthanized. First, $3 \%$ pentobarbital sodium was intraperitoneally injected at a dosage of $60 \mathrm{mg} / \mathrm{kg}$ and when the righting reflex disappeared, rats were decapitated. The brain was taken out and rapidly frozen at $-80^{\circ} \mathrm{C}$.

For testing for NE and 5-HT, a standard curve was recorded and analysis was performed using an Acquity Ultra-Performance Liquid Chromatography/SynaptG2 Quadrupole Time-of-Flight mass spectrometer (Waters China, Ltd.). The entire brain was collected for amino acid detection. Analysis of Asp, GABA and Glu was performed using the Agilent 1260 Infinity system (Agilent Technologies, Inc.) coupled with a Nova-Pak ${ }^{\circledR} \mathrm{C} 18$ (5 $\mu \mathrm{m})$ column (Waters China, Ltd). The chromatographic analysis method used was that described in the AccQ-Tag amino acid chemical reagent package (Waters China, Ltd.). The Waters AccQ-Tag amino acid chemical reagent package contains Amino acid derivatization reagent, Nova-Pak ${ }^{\circledR} \mathrm{C} 18$ $(5 \mu \mathrm{m})$ column, amino acid standards and an instruction of operation and analysis.

ELISA for CGRP and SP. At 150 min after modeling, six rats from each group were anesthetized with $1.5 \%$ isoflurane. Blood samples were collected from the abdominal aorta. Subsequently, CGRP and SP were detected using the ELISA kits according to the manufacturer's protocols.

Immunohistochemical analysis. Immunohistochemical staining of the brain stem was performed using a previously described method (34-36). The brain stem was fixed with fresh $4 \%$ paraformaldehyde solution. Paraffin-embedded sections with a thickness of $4 \mu \mathrm{m}$ were then prepared, where the slices were deparaffinized and rehydrated using a decreasing ethanol 
(1)<smiles>O=c1cc(-c2ccc(O)c(O)c2)oc2cc(O)cc(O)c12</smiles>

(2)<smiles>COc1cc2oc(-c3ccc(O)c(O)c3)c(OC)c(=O)c2c(O)c1OC</smiles>

(3)<smiles>COC1=CC2(OC(c3ccc(OC)c(O)c3)C(OC)C(=O)C2OC)C(OC)C(O)=C1OC</smiles>

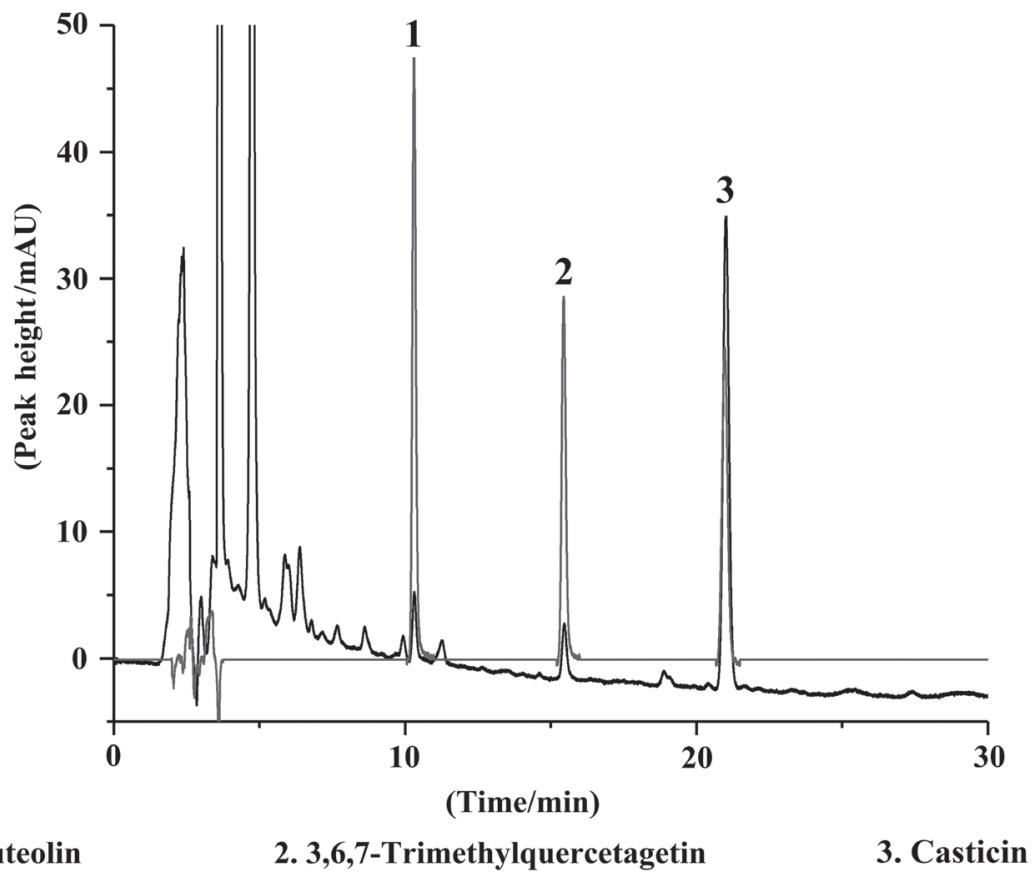

Figure 1. Quality control of VME using three reference substances. Peak 1, luteolin; peak 2, 3,6,7-trimethylquercetagetin and peak 3, casticin.

gradient before being blocked with $10 \%$ normal goat serum at room temperature for $1 \mathrm{~h}$. The tissue sections were incubated with rabbit anti-c-fos primary antibody (1:50) at $4^{\circ} \mathrm{C}$ for $48 \mathrm{~h}$ before incubation with goat anti-rabbit secondary antibody (1:200) at $25^{\circ} \mathrm{C}$ for $1 \mathrm{~h}$. Then nuclei were counterstained with hematoxylin at room temperature for $2 \mathrm{~min}$. The number of c-fos-immunoreactive cells (c-fos IR cells) was determined.

Statistical analysis. Values are expressed as the mean \pm standard deviation. Each group of data was analyzed using SPSS 25 software (IBM Corp.). Kolmogorov-Smirnov was used to verify whether the data conformed to the normal distribution. Data with a normal distribution were compared using one-way analysis of variance followed by Tukey's post-hoc test, $\mathrm{P}<0.05$ or $\mathrm{P}<0.01$ were considered to indicate statistically significant differences. Statistical analyses of the results were performed out in a double-blinded fashion.

\section{Results}

Quality control of VME. Luteolin, 3,6,7-trimethylquercetagetin and casticin are characteristic components of Fructus Viticis and were detected by HPLC. A chromatogram of the extracts and reference compounds is provided in Fig. 1. All three compounds were detected in the VME and the content of casticin met the Chinese Pharmacopoeia standard of casticin $>0.03 \%$ (37).

Analgesic effect of VME. The results of the hot plate test are provided in Fig. 2, indicating that the morphine significantly decreased the pain threshold at 30, 60 and 90 min $(\mathrm{P}<0.05)$, and the high dose of VME decreased the pain threshold slightly at the same time points, but no significance was observed $(\mathrm{P}>0.05)$. As depicted in Fig. 3, the acetic acid-induced writhing test indicated that the aspirin obviously reduced the pain latency and the number of times of writhing $(\mathrm{P}<0.01$ for each). In addition, the high dose VME reduced the pain latency $(\mathrm{P}<0.05)$ and the number of times of writhing $(\mathrm{P}<0.05)$.

Effect of VME on migraine-like behaviour. The rats of the model group had migraine-like responses after $5 \mathrm{~min}$ of nitroglycerin injection, including the use of fore- or hind paws to scratch the head and development of ear redness. There was no ear redness in the control group after subcutaneous injection of normal saline and within 120 min, only one or two episodes of head scratching and a few incidents of cage climbing were observed (Fig. 4), which may have been at random. Compared with the model group, there were significant differences regarding these parameters $(\mathrm{P}<0.01$ for each). The rats in the model group exhibited shorter latencies of ear redness, as well as frequent head scratching and cage climbing, with an increased amount of discomfort-associated behaviour and a modeling success rate of $100 \%(\mathrm{P}<0.01)$. The latency of ear redness in the rizatriptan and VME high-dose group was significantly higher compared with that in the model group ( $\mathrm{P}<0.01$; Fig. 4A). The amount of head scratching and cage climbing was also significantly lower than that of the model group ( $\mathrm{P}<0.01$; Fig. $4 \mathrm{~B}$ and $\mathrm{C})$. The effect in the VME low-dose group compared with the trend in the model group was not statistically significant. 


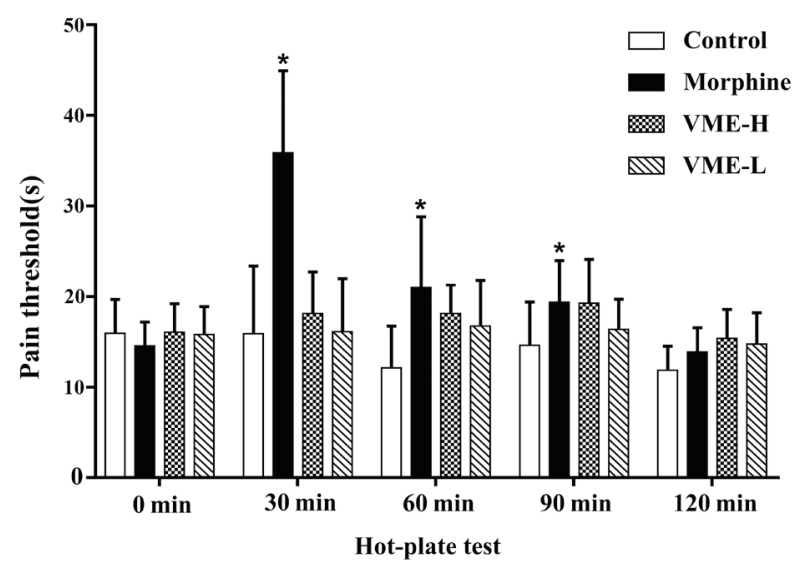

Figure 2. Effect of VME on the pain threshold of mice subjected to hot plate stimulus. Values are expressed as the mean \pm standard deviation $(n=8)$. ${ }^{*} \mathrm{P}<0.05$ vs. control group. VME-H/L, high/low concentration of VME.
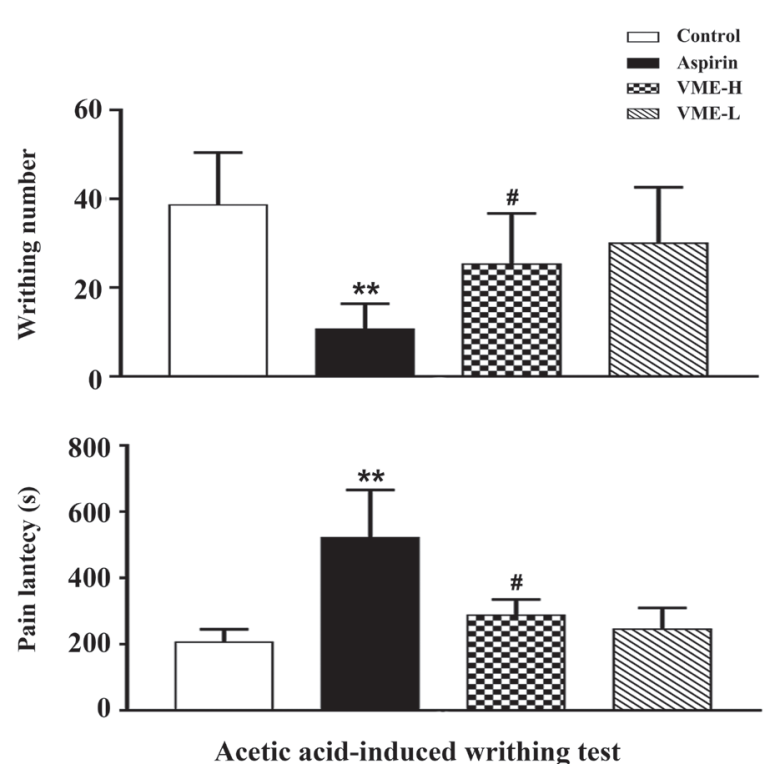

Figure 3. Effect of VME on pain latency and number of times of writhing of mice caused by the acetic acid stimulus. Values are expressed as the mean \pm standard deviation $(\mathrm{n}=8)$. ${ }^{* *} \mathrm{P}<0.01$ vs. control group; ${ }^{\#} \mathrm{P}<0.05$ vs. model group. VME-H/L, high/low concentration of VME.

Effect of VME on neurotransmitters and amino acids in the rat model of migraine. As presented in Fig. 5, the effect of the test drugs on various neurotransmitters in the brain of rats with migraine was assessed. The 5-HT levels in the model group were lower than those in the control group $(\mathrm{P}<0.05)$, while pre-treatment with rizatriptan and VME at the high dose inhibited this phenomenon $(\mathrm{P}<0.01$; $\mathrm{P}<0.05$, Fig. $5 \mathrm{~A})$. Furthermore, subcutaneous injection of nitroglycerin reduced the level of NE, while this was prevented by pre-treatment with high-dose rizatriptan and VME $(\mathrm{P}<0.01$, for each; Fig. $5 \mathrm{~B})$. The low dose of VME had no significant effect on rats with migraine $(\mathrm{P}>0.05)$.

The amino acid contents are provided in Fig. 5C-E. The Asp and Glu levels were increased following subcutaneous injection of nitroglycerin $(\mathrm{P}<0.01)$, which was significantly inhibited by pre-treatment with high-dose VME $(\mathrm{P}<0.05$ and $\mathrm{P}<0.01$, respectively), which was similar to the effect of rizatriptan $(\mathrm{P}<0.01$ and $\mathrm{P}<0.05$, respectively). The levels of GABA were decreased in the model group $(\mathrm{P}<0.05)$, and in comparison, they were significantly increased in the rizatriptan and high-dose VME groups $(\mathrm{P}<0.01)$.

Effect of VME on SP and CGRP in the rat model of migraine. The levels of SP and CGRP are provided in Fig. 6A and B, respectively. The levels of SP and CGRP were increased following subcutaneous injection of nitroglycerin $(\mathrm{P}<0.01$ and $\mathrm{P}<0.05$, respectively), which was significantly inhibited by pre-treatment high-dose VME $(\mathrm{P}<0.05)$. There was no significant difference in the SP and CGRP content between the high-dose VME and control groups $(\mathrm{P}>0.05)$.

Effect of VME on c-fos expression in brain stem neurons of the rat model of migraine. $\mathrm{C}$-fos-positive staining in the brain stem neurons was assessed as a central pain sensory marker. The number of c-fos IR cells in the rats of different groups was determined (Fig. 7). In the migraine model group, the number of c-fos IR cells was significantly higher than that of the control group $(\mathrm{P}<0.01)$. The number of $\mathrm{c}$-fos IR cells in the brain stem of the VME high-dose group, the rizatriptan and VME low-dose groups were significantly lower than that in the model group $(\mathrm{P}<0.01$ or $\mathrm{P}<0.05)$, indicating that $\mathrm{VME}$ reduced the migraine-associated expression of $\mathrm{c}-\mathrm{fos}$ in the brain stem.

\section{Discussion}

Migraine attacks seriously affect the lives of patients. During the onset process of migraine, patients experience irritability (38). Several studies indicated that nitroglycerin administration in rodents may be used to generate a model to resemble migraine in humans $(13,39)$. After intraperitoneal injection of nitroglycerin, the level of $\alpha$-amino-3-hydroxy- 5 -methyl-4-isoxazolepropionic acid (AMPA) receptor and the phosphorylation of glutamate A1 at Ser831 in the posterior spinal trigeminal nerve were reported to be increased and central sensitization was enhanced (40). Nitroglycerin, as a nitric oxide promoter (41), activates the TMVS and induces hyperalgesia in rats. Model rats exhibit similar pain responses to those of patients with migraine, and associated signs/behaviours in those rats include ear redness, head scratching and cage climbing. Therefore, behavioural testing may be used to assess the level of migraine pain in rats. In the present study, the behavioural tests indicated that rats in the model group exhibited characteristics of restlessness, with an increased latency of ear redness, head scratching and cage climbing, whereas pre-treatment with Fructus Viticis alleviated the migraine-like headache response. Thus, the rat model of migraine was successfully established. According to different requirements, a variety of migraine models have been previously established, and for each model, it should be determined whether it was successfully confirmed based on the corresponding behavioural criteria. In addition to the migraine model induced by subcutaneous injection of nitroglycerin, further examples include the dural electrical stimulation model and the electrophysiology chemical provocation model (42-44). 

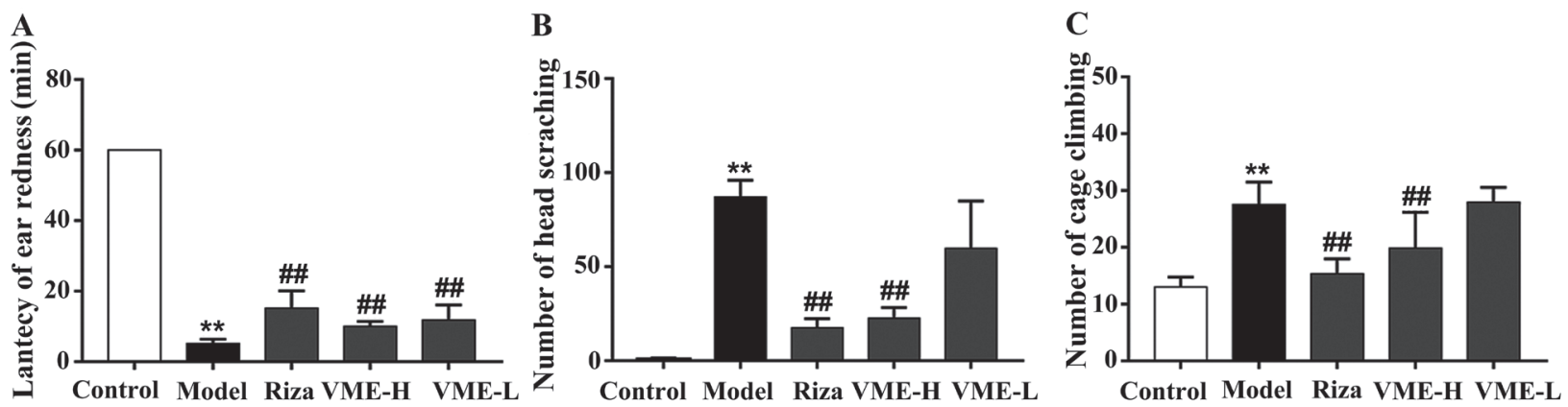

Figure 4. Effect of VME on (A) latency of red ears, (B) number of head scratching and (C) number of cage climbing following subcutaneous injection of nitroglycerin. Values are expressed as the mean \pm standard deviation $(\mathrm{n}=12) .{ }^{* *} \mathrm{P}<0.01$ vs. control group; ${ }^{\# \#} \mathrm{P}<0.01$ vs. model group. Riza, rizatriptan benzoate; VME-H/L, high/low concentration of VME.
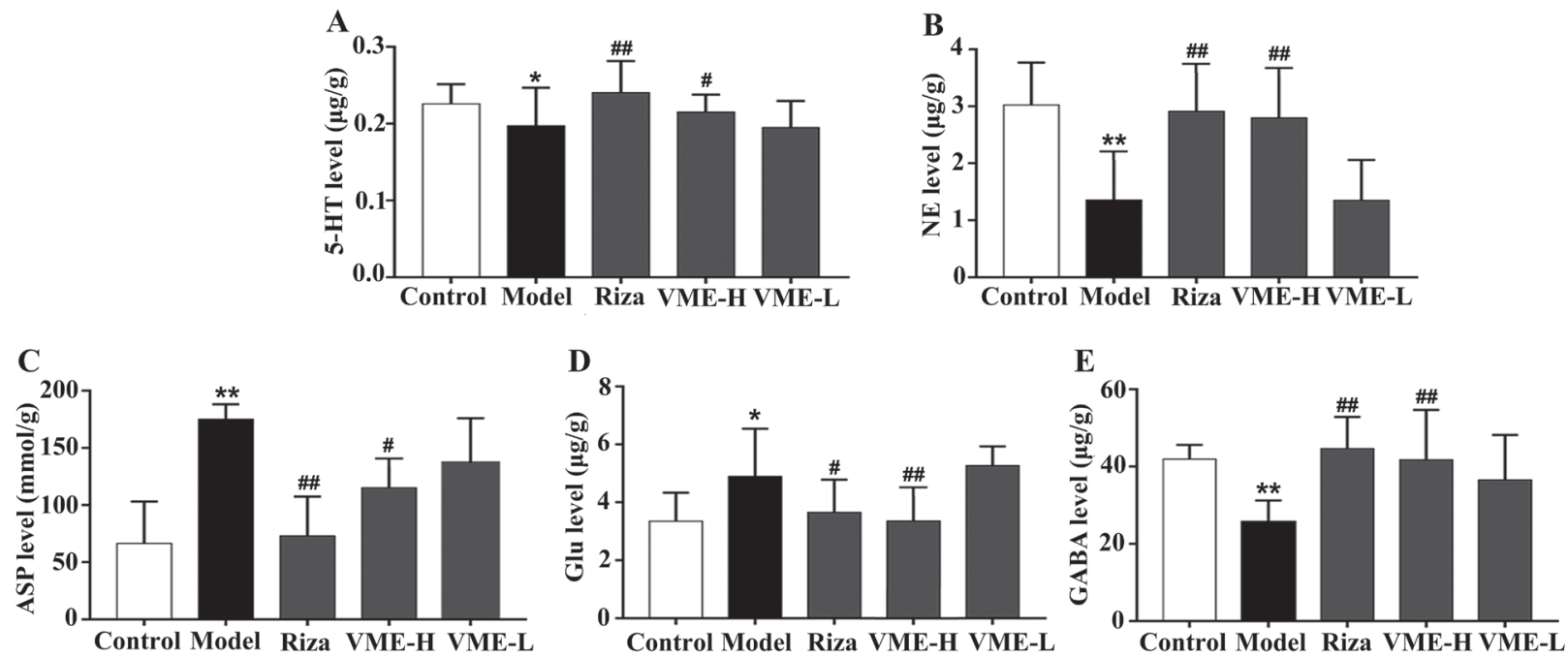

Figure 5. Effect of VME on the levels of (A) 5-hydroxytryptamine, (B) NE, (C) ASP, (D) Glu and (E) GABA induced by subcutaneous injection of nitroglycerin. Values are expressed as the mean \pm standard deviation $(\mathrm{n}=6) .{ }^{*} \mathrm{P}<0.05,{ }^{* *} \mathrm{P}<0.01$ vs. control group; ${ }^{\#} \mathrm{P}<0.05,{ }^{\# \#} \mathrm{P}<0.01$ vs. model group. Riza, rizatriptan benzoate; VME-H/L, high/low concentration of VME; NE, norepinephrine; ASP, aspartic acid; Glu, glutamic acid; GABA, $\gamma$-aminobutyric acid.

A

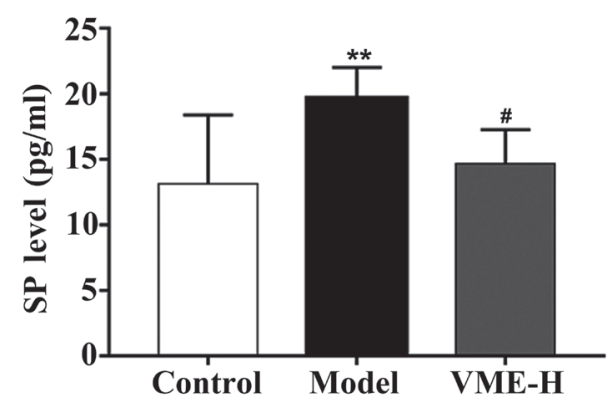

B

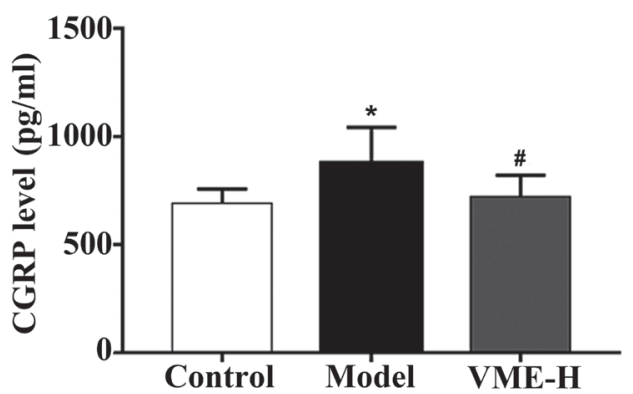

Figure 6. Effect of VME on the levels of (A) SP and (B) CGRP induced by subcutaneous injection of nitroglycerin. Values are expressed as the mean \pm standard deviation ( $\mathrm{n}=6$ ). ${ }^{*} \mathrm{P}<0.05,{ }^{* *} \mathrm{P}<0.01$ vs. control group; $\mathrm{P}<0.05$, vs. model group. Riza, rizatriptan benzoate; VME-H, high concentration of VME; SP, substance P; CGRP, calcitonin gene-related peptide.

NE and 5-HT are neurotransmitters, the downregulation of which are associated with migraine; consequently, they are widely applied as an auxiliary parameter in the diagnosis of migraine (43). Subcutaneous injection of nitroglycerin as an external stimulus may stimulate excitatory signaling in the TMVS. NE and 5-HT are critical regulatory factors and may inhibit transcription of the CGRP gene and prevent CGRP release to decrease the level of CGRP. Drugs that reduce CGRP have been used in the clinic to alleviate migraine (45). Simultaneously, the two neurotransmitters are able to increase the binding of GABA to glycine neurons and $\alpha 25$-HT receptors to reduce the damaging effect of external stimuli to the 

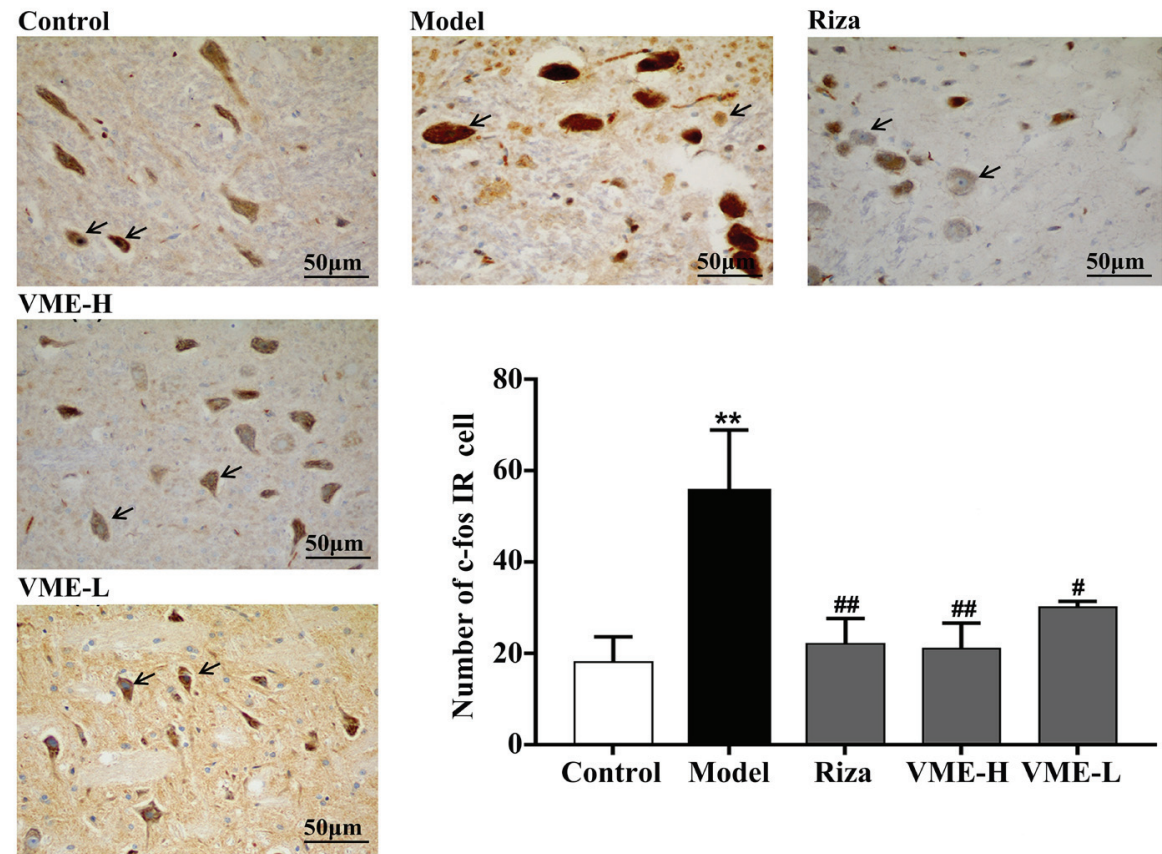

Figure 7. Effect of VME on the amount of c-fos IR cells in the brain stem induced by subcutaneous injection of nitroglycerin. Representative histology images are provided (arrows indicate immunopositive cells; scale bar, $50 \mu \mathrm{m}$ ). Values are expressed as the mean \pm standard deviation $(\mathrm{n}=6) .{ }^{* *} \mathrm{P}<0.01 \mathrm{vs}$. control group; ${ }^{\#} \mathrm{P}<0.05,{ }^{\# /} \mathrm{P}<0.01$ vs. model group. IR, immunoreactive; Riza, rizatriptan benzoate; VME-H/L, high/low concentration of VME.

TMVS (46). In the present study, when compared to the model group, the 5-HT and NE levels of rats in the high-dose VME groups were increased.

The trigeminal nucleus is where the cell bodies of the trigeminal nerve are aggregated in the brain stem. It is the largest cranial nucleus, spanning the entire brain stem and extending from the midbrain to the medulla. SP and CGRP are vasoactive neuropeptides released by activated peripheral nociceptive sensory nerve terminals (47). It is generally recognized that migraine headaches result from activation and sensitization of trigeminal sensory afferent fibers, leading to the release of neuropeptides including CGRP and SP. This triggers an inflammatory cascade that causes neurogenic inflammation (48). In response to prolonged TMVS, SP and CGRP are released from the trigeminal sensory nerves in the area of the dural blood vessels, leading to endothelium-dependent vasodilation, increased microvascular permeability, as well as subsequent plasma and protein extravasation, causing neuroinflammation. SP is the prime mediator, via neurokinin (NK)-1 receptors, of plasma leakage at the site of inflammation (49,50), whereas CGRP and SP induce vasodilatation. The involvement of SP in plasma leakage and neurogenic inflammation has been well established by the ability of NK-1 receptor antagonists and SP immunoneutralization to attenuate neurogenic exudative responses to a variety of stimuli (51). A significant increase in plasma SP and CGRP levels has been demonstrated during the headache phase of migraine.

In the present study, VME was indicated to have definite analgesic effects, making it apt for migraine treatment. The mechanism for this is likely linked to the regulation of abnormal contents of amino acids and neurotransmitters and the reduction of SP, CGRP and positive expression of c-fos in the brain stem. There is an accumulation of evidence demonstrating that numerous traditional Chinese medicines, which are widely used for treating various neurological headaches, confer beneficial and reliable therapeutic effects (52-54). Fructus Viticis is a common prescription in Traditional Chinese Medicine. It has been reported that $25 \%$ percent of Fructus Viticis use is for clinical purposes (24). In addition, Fructus Viticis is an ideal drug for migraine prevention for three reasons: First, casticin, the major active component of Fructus Viticis, is anti-inflammatory and inhibits nitrous oxide production in cells (55). Luteolin is another flavone of Fructus Viticis and is able to ameliorate hyperalgesia in the central nervous system (56). A previous study demonstrated that luteolin has anti-nociceptive effects on acute thermal pain and persistent inflammatory pain in rodents (57). Previous research has also suggested that luteolin displays anxiolytic and sedative effects through interaction with benzodiazepine binding sites (58). Finally, Fructus Viticis is a traditional medicine that is widely used in Asian countries, including China, Korea and Japan, to treat migraine, headache and inflammation $(57,59)$.

Data from the present study suggest that the methanolic extract of Fructus Viticis may be used to treat disturbances of amino acid metabolism. During the migraine aura and headache phase, amino acid levels are abnormal. When the harmful signal is generated, extreme depolarization of glial and neuronal cell membranes results in disruption of ionic gradients, a rise in extracellular potassium concentrations and release of glutamate (12). Furthermore, the release of neurotransmitter GABA may inhibit transmission of nerve signaling, modulate pain thresholds and produce analgesic effects (60-62). These two different types of amino acid have opposite functions. The present results indicated that high-dose and low-dose VME significantly increased GABA levels in rats. Furthermore, SP, Glu and Asp have been proven to mediate the transmission of excitatory signals to the synapse $(63,64)$. In clinical studies 
on migraine patients, elevated levels of Glu and Asp were detected in the cerebrospinal fluid (65). The balance between the two types of neurotransmitter provides a framework in which other factors regulate functions of the human brain (66). The experimental results of the present study indicated that the content of Asp and Glu was high in the model group and decreased in the drug treatment groups.

The present results indicated that methanolic extract of Fructus Viticis alleviated pain-like behavior in a rat models of migraine, reduced pain sensitization of the trigeminal nerve and decreased the expression of c-fos. c-fos protein, encoded by the c-fos gene, is often used to evaluate nervous activity (67). Subcutaneous injection of nitroglycerin in rats may lead to elevation of CGRP (9), which in turn is able to induce the expression of the c-fos gene in the trigeminal nucleus caudalis (68). As indicated in a previous study, the amount of c-fos-expressing neurons is increased in the brain stem nuclei of rats with migraine (11). C-fos is commonly accepted as a neural marker of pain in the central nervous system. Upon apparent hyperalgesic sensitization of the central nervous system, c-fos protein is expressed. Therefore, the amount of c-fos IR neurons may, to a certain degree, determine the extent of migraine. Due to its immediacy, it may be assumed that c-fos is one of the earliest indicators of successful migraine modeling. However, this is nonspecific, as c-fos is also expressed during tumorigenesis as a subcomponent of the macromolecular activator protein 1 complex (69), which is associated with neoplastic transformation and progression. For instance, it was reported that c-fos has a role in endometrial, cervical and colorectal cancer $(70,71)$.

In conclusion, the present study demonstrated that VME has an analgesic effect in mice and is able to mitigate the migraine-like pain response to nitroglycerin in model rats. CGRP and SP, as vasoactive neuropeptides released by activated peripheral nociceptive sensory nerve terminals during migraine, were reduced in the group treated with high-dose VME. The therapeutic efficacy is likely a result of the inhibitory effect on the transmission of trigeminal nerve injury signals. The present study provides a further scientific basis for the anti-migraine effects of Fructus Viticis.

\section{Acknowledgements}

The authors gratefully acknowledge the provided the hot plate and other experimental facilities of the Institute of Material Medica Integration and Transformation for Brain Disorders (Chengdu, China).

\section{Funding}

This study was supported by the National Nature Science Foundation of China (grant no. 81303082) and Major R\&D (Major Projects) Projects in Sichuan Province (grant no. 19ZDYF0600).

\section{Availability of data and materials}

The datasets used and/or analyzed during the current study are available from teh corresponding author on reasonable request.

\section{Authors' contributions}

SX and KF conceived the project, WW, TP and LQ performed the experiments. HC performed data analysis. JW supervised the project. WW drafted and revised the manuscript.

\section{Ethics approval and consent to participate}

The protocols were approved by the animal experimental ethics committee of Ethics Committee for Animal Experiments of the Institute of Materia Medica Integration and Transformation for Brain Disorders (grant no. 20131017; Chengdu, China).

\section{Patient consent for publication}

Not applicable.

\section{Competing interests}

The authors declare that they have no competing interests.

\section{References}

1. GBD 2015 Neurological Disorders Collaborator Group: Global, regional, and national burden of neurological disorders during 1990-2015: A systematic analysis for the Global Burden of Disease Study 2015. Lancet Neurol 16: 877-897, 2017.

2. Patton GC, Olsson CA, Skirbekk V, Saffery R, Wlodek ME, Azzopardi PS, Stonawski M, Rasmussen B, Spry E, Francis K, et al: Adolescence and the next generation. Nature 554: 458-466. 2018.

3. Mawet J, Kurth T and Ayata C: Migraine and stroke: In search of shared mechanisms. Cephalalgia 35: 165-181, 2015.

4. Bolay H, Reuter U, Dunn AK, Huang Z, Boas DA and Moskowitz MA: Intrinsic brain activity triggers trigeminal meningeal afferents in a migraine model. Nat Med 8: 136-142, 2002.

5. Asghar MS, Hansen AE, Larsson HB, Olesen J and Ashina M: Effect of CGRP and sumatriptan on the BOLD response in visual cortex. J Headache Pain 13: 159-166, 2012.

6. Iyengar S, Ossipov MH and Johnson KW: The role of calcitonin gene-related peptide in peripheral and central pain mechanisms including migraine. Pain 158: 543-559, 2017.

7. Miyoshi $\mathrm{H}$ and Nakaya Y: Calcitonin gene-related peptide activates the $\mathrm{K}+$ channels of vascular smooth muscle cells via adenylate cyclase. Basic Res Cardiol 90: 332-336, 1995.

8. Basbaum AI, Bautista DM, Scherrer G and Julius D: Cellular and molecular mechanisms of pain. Cell 139: 267-284, 2009.

9. Demartini C, Tassorelli C, Zanaboni AM, Tonsi G, Francesconi O, Nativi $C$ and Greco R: The role of the transient receptor potential ankyrin type-1 (TRPA1) channel in migraine pain: Evaluation in an animal model. J Headache Pain 18: 94, 2017.

10. Borkum JM: Migraine triggers and oxidative stress: A narrative review and synthesis. Headache 56: 12-35, 2016.

11. Bohár Z, Fejes-Szabó A, Tar L, Varga H, Tajti J, Párdutz Á and Vécsei L: Evaluation of c-Fos immunoreactivity in the rat brainstem nuclei relevant in migraine pathogenesis after electrical stimulation of the trigeminal ganglion. Neurol Sci 34: 1597-1604, 2013.

12. Dodick DW, Migraine. Lancet 391: 1315-1330, 2018.

13. Moye LS and Pradhan AAA: Animal model of chronic migraine-associated pain. Curr Protoc Neurosci 80: 9.60.1-9.60.9, 2017.

14. Rains JC, Penzien DB, McCrory DC and Gray RN: Behavioral headache treatment: History, review of the empirical literature, and methodological critique. Headache 45 (45 Suppl): S92-S109, 2005.

15. Gallagher RM and Kunkel R: Migraine medication attributes important for patient compliance: Concerns about side effects may delay treatment. Headache 43: 36-43, 2003. 
16. Chen DQ, Cao G, Chen H, Argyopoulos CP, Yu H, Su W, Chen L, Samuels DC, Zhuang S, Bayliss GP, et al: Identification of serum metabolites associating with chronic kidney disease progression and anti-fibrotic effect of 5-methoxytryptophan. Nat Commun 10: 1476, 2019.

17. Chen DQ, Feng YL, Cao G and Zhao YY: Natural products as a source for antifibrosis therapy. Trends Pharmacol Sci 39: 937-952, 2018.

18. Zhao L, Chen J,Li Y, Sun X, Chang X, Zheng H, Gong B, Huang Y, Yang M, Wu X, et al: The Long-term effect of acupuncture for migraine prophylaxis: A randomized clinical trial. JAMA Intern Med 177: 508-515, 2017.

19. Sun YY, Zhang WJ, Dong CL, Zhang XF, Ji J, Wang X, Wang L, Hu WL, Du WJ, Cui CL, et al: Baicalin alleviates Nitroglycerin-induced migraine in rats via the trigeminovascular system. Phytother Res 31: 899-905, 2017.

20. Wu Y,Pan X, Xu Y, Lu X, He S, He R and Gong M: Optimization of combinations of ginsenoside-Rg1, ginsenoside-Rb1, evodiamine and rutaecarpine for effective therapy of mouse migraine. J Nat Med 70: 207-216, 2016.

21. Hou M, Tang Q, Xue Q, Zhang X, Liu Y, Yang S, Chen L and $\mathrm{Xu}$ X: Pharmacodynamic action and mechanism of Du Liang soft capsule, a traditional Chinese medicine capsule, on treating nitroglycerin-induced migraine. J Ethnopharmacol 195: 231-237, 2017.

22. Hu Y, Xin HL, Zhang QY, Zheng HC, Rahman K and Qin LP: Anti-nociceptive and anti-hyperprolactinemia activities of Fructus Viticis and its effective fractions and chemical constituents. Phytomedicine 14: 668-674, 2007.

23. Guan R, Wang D, Yu Z, Wang X and Lan T: Preparative isolation and purification of the active components from Viticis Fructus by high-speed counter-current chromatography. Se $\mathrm{Pu} 28$ : 1043-1047, 2010 (In Chinese).

24. Yan YY, Dai JZ, Yang CX and Zhang GM: Analysis of traditional Chinese medicine syndromes in 159 German migraine patients. Zhongguo Zhong Yao Za Zhi 42: 2599-2605, 2017 (In Chinese).

25. Liu P, Xing B, Chu Z, Liu F, Lei G, Zhu L, Gao Y, Chen T and Dang YH: Dopamine D3 receptor knockout mice exhibitabnormal nociception in a sex-different manner. J Neurosci Res 95 $1438-1445,2017$

26. Wang G, Hu Z, Song X, Cui Q, Fu Q, Jia R, Zou Y, Li L and Yin Z: Analgesic and Anti-inflammatory activities of resveratrol through classic models in mice and rats. Evid Based Complement Alternat Med 2017: 5197567, 2017

27. Zakaria A, Jais MR and Ishak R: Analgesic properties of nigella sativa and eucheuma cottonii extracts. J Nat Sci Biol Med 9: 23-26, 2018.

28. Nair AB and Jacob S: A simple practice guide for dose conversion between animals and human. J Basic Clin Pharm 7: 27-31, 2016.

29. Fu W, Dai Y, Ma T, Wei J, Chen H and Xu S: Tongluo Xingnao effervescent tablet reverses memory deficit and reduces plaque load in APPswe/PS1dE9 mice. Exp Ther Med 15: 4005-4013, 2018.

30. Burstein R, Noseda R and Borsook D: Migraine: Multiple processes, complex pathophysiology. J Neurosci 35: 6619-6629, 2015.

31. Pradhan AA, Smith ML, McGuire B, Tarash I, Evans CJ and Charles A: Characterization of a novel model of chronic migraine. Pain 155: 269-274, 2014

32. Tang Y, Liu S, Shu H, Xing Y and Tao F: AMPA receptor GluA1 Ser831 phosphorylation is critical for nitroglycerin-induced migraine-like pain. Neuropharmacology 133: 462-469, 2018.

33. Ben Aissa M, Tipton AF, Bertels Z, Gandhi R, Moye LS, Novack M, Bennett BM, Wang Y, Litosh V, Lee SH, et al: Soluble guanylyl cyclase is a critical regulator of migraine-associated pain. Cephalalgia 38: 1471-1484, 2018.

34. Becerra L, Bishop J, Barmettler G, Kainz V, Burstein R and Borsook D: Brain network alterations in the inflammatory soup animal model of migraine. Brain Res 1660: 36-46, 2017.

35. Munro G, Jansen-Olesen I and Olesen J: Animal models of pain and migraine in drug discovery. Drug Discov Today 22: $1103-1111,2017$

36. D'Andrea G and Leon A: Pathogenesis of migraine: From neurotransmitters to neuromodulators and beyond. Neurol Sci 31 (Suppl 1): S1-S7, 2010.

37. State Pharmacopoeia Committee. Pharmacopoeia of the People's Republic of China. Part I. Beijing: China Medical Science and Technology Press, 2015.
38. Silberstein SD, Dodick DW, Bigal ME, Yeung PP, Goadsby PJ, Blankenbiller T, Grozinski-Wolff M, Yang R, Ma Y and Aycardi E: Fremanezumab for the preventive treatment of chronic migraine. N Engl J Med 377: 2113-2122, 2017.

39. Akerman S, Holland PR, Lasalandra MP and Goadsby PJ: Endocannabinoids in the brainstem modulate dural trigeminovascular nociceptive traffic via CB1 and 'triptan' receptors: Implications in migraine. J Neurosci 33: 14869-14877, 2013.

40. Malhotra R: Understanding migraine: Potential role of neurogenic inflammation. Ann Indian Acad Neurol 19: 175-182, 2016.

41. Martins LB, Teixeira AL and Domingues RB: Neurotrophins and migraine. Vitam Horm 104: 459-473, 2017.

42. Rosa AC and Fantozzi R: The role of histamine in neurogenic inflammation. Br J Pharmacol 170: 38-45, 2013.

43. Williamson DJ, Hargreaves RJ, Hill RG and Shepheard SL: Intravital microscope studies on the effects of neurokinin agonists and calcitonin gene-related peptide on dural vessel diameter in the anaesthetized rat. Cephalalgia 17: 518-524, 1997.

44. Alvaro G and Di Fabio R: Neurokinin 1 receptor antagonists-current prospects. Curr Opin Drug Discov Devel 10: 613-621, 2007.

45. Zhou L, Pinyi C, Ling L, Zhang Y, Liu X, Wu Y, Jiang L, Cheng D, Huang W, Pettigrew JC and Yi D: Systematic review and meta-analysis of traditional Chinese medicine in the treatment of migraines. Am J Chin Med 41: 1011-1025, 2013.

46. Hu HQ, Zhou YH and Wang XL: Clinical study on effect of Xiaoyao Nose Drops in stopping episode of Migraine. Chin J Integr Med 12: 112-117, 2006.

47. Li JC, Shen XF, Meng XL, Zhang Y and Lai XR: Analgesic effect and mechanism of the three TCM-herbal drug-combination Tou Feng Yu pill on treatment of migraine. Phytomedicine 18: 788-794, 2011.

48. Song HM, Park GH, Park SB, Kim HS, Son HJ, Um Y and Jeong JB: Vitex rotundifolia Fruit Suppresses the proliferation of human colorectal cancer cells through Down-regulation of Cyclin D1 and CDK4 via proteasomal-dependent degradation and transcriptional inhibition. Am J Chin Med 46: 191-207, 2018.

49. Hara K, Haranishi Y, Terada T, Takahashi Y, Nakamura M and Sata T: Effects of intrathecal and intracerebroventricular administration of luteolin in a rat neuropathic pain model. Pharmacol Biochem Behav 125: 78-84, 2014.

50. Backhouse N, Delporte C, Apablaza C, Farías M, Goïty L, Arrau S, Negrete R, Castro C and Miranda H: Antinociceptive activity of Buddleja globosa (matico) in several models of pain. J Ethnopharmacol 119: 160-165, 2008.

51. Ishisaka M,Kakefuda K, Yamauchi M, Tsuruma K, Shimazawa M, Tsuruta A and Hara H: Luteolin shows an antidepressant-like effect via suppressing endoplasmic reticulum stress. Biol Pharm Bull 34: 1481-1486, 2011.

52. Lee C, Lee JW, Jin Q, Lee HJ, Lee SJ, Lee D, Lee MK, Lee CK, Hong JT, Lee MK and Hwang BY: Anti-inflammatory constituents from the fruits of Vitex rotundifolia. Bioorg Med Chem Lett 23: 6010-6014, 2013

53. Ambrosini A, Di Lorenzo C, Coppola G and Pierelli F: Use of Vitex agnus-castus in migrainous women with premenstrual syndrome: An open-label clinical observation. Acta Neurol Belg 113: 25-29, 2013

54. Noseda R, Borsook D and Burstein R: Neuropeptides and neurotransmitters that modulate thalamo-cortical pathways relevant to migraine headache. Headache 57 (Suppl 2): S97-S111, 2017.

55. Andreou AP, Shields KG and Goadsby PJ: GABA and valproate modulate trigeminovascular nociceptive transmission in the thalamus. Neurobiol Dis 37: 314-323, 2010.

56. Janssen SP, Truin M, Van Kleef M and Joosten EA: Differential GABAergic disinhibition during the development of painful peripheral neuropathy. Neuroscience 184: 183-194, 2011.

57. Zhu X, Han Y, Xiong W, Liu W, Lu S, Li J, Wang H and Fan Z: Effects of heating coagulation of middle meningeal artery on plasma CGRP level and c-fos expression in migraine rat triggered by nitroglycerin. Neurol Sci 32: 589-594, 2011.

58. van IJzendoorn DGP, Forghany Z, Liebelt F, Vertegaal AC, Jochemsen AG, Bovée JVMG, Szuhai K and Baker DA: Functional analyses of a human vascular tumor FOS variant identify a novel degradation mechanism and a link to tumorigenesis. J Biol Chem 292: 21282-21290, 2017.

59. Wang YH, Liang S, Xu DS, Lin X, He CY, Feng Y and Hong YL: Effect and mechanism of senkyunolide I as an anti-migraine compound from Ligusticum chuanxiong. J Pharm Pharmacol 63: 261-266, 2011 
60. Tassorelli $\mathrm{C}$ and Joseph SA: Systemic nitroglycerin induces Fos immunoreactivity in brainstem and forebrain structures of the rat. Brain Res 682: 167-181, 1995.

61. Pan X, Wang M, Wu Y, Lu X, Shang Y, Xu Y, Zhai Y, Li J, Li Z and Gong M: Identification of active ingredients in Wuzhuyu decoction improving migraine in mice by spectral efficiency association. Mol Med Rep 12: 1524-1534, 2015.

62. Zhang ZH, Vaziri ND, Wei F, Cheng XL, Bai X and Zhao YY: An integrated lipidomics and metabolomics reveal nephroprotective effect and biochemical mechanism of Rheum officinale in chronic renal failure. Sci Rep 6: 22151, 2016.

63. Zhang Q, Zhao Y, Guo Y, Cao DY, Tian YL, Yao FR and Wang HS: Electrophysiological evidence for the interaction of substance $\mathrm{P}$ and glutamate on Adelta and $\mathrm{C}$ afferent fibre activity in rat hairy skin. Clin Exp Pharmacol Physiol 33: 1128-1133, 2007.

64. Gundersen V, Chaudhry FA, Bjaalie JG, Fonnum F, Ottersen OP and Storm-Mathisen J: Synaptic vesicular localization and exocytosis of L-aspartate in excitatory nerve terminals: A quantitative immunogold analysis in rat hippocampus. J Neurosci 18: 6059-6070, 1998.

65. Vikelis M and Mitsikostas DD: The role of glutamate and its receptors in migraine. CNS Neurol Disord Drug Targets 6: 251-257, 2007.

66. Gao R and Penzes P: Common mechanisms of excitatory and inhibitory imbalance in schizophrenia and autism spectrum disorders. Curr Mol Med 15: 146-167, 2015.
67. Young D, Ma J, Cherkerzian S, Froimowitz MP, Ennulat DJ, Cohen BM, Evans ML and Lange N: Automated identification of Fos expression. Biostatistics 3: 351-364, 2001.

68. Chen DQ, Chen H, Chen L, Vaziri ND, Wang M, Li XR and Zhao YY: The link between phenotype and fatty acid metabolism in advanced chronic kidney disease. Nephrol Dial Transplant 32: 1154-1166, 2017.

69. Dou F, Miao H, Wang JW, Chen L, Wang M, Chen H, Wen AD and Zhao YY: An integrated lipidomics and phenotype study reveals protective effect and biochemical mechanism of traditionally used Alisma orientale Juzepzuk in chronic renal disease. Front Pharmacol 9: 53, 2018.

70. Pandey MK, Liu G, Cooper TK and Mulder KM: Knockdown of c-Fos suppresses the growth of human colon carcinoma cells in athymic mice. Int J Cancer 130: 213-222, 2012.

71. Lu C, Shen Q, DuPré E, Kim H, Hilsenbeck S and Brown PH: cFos is critical for MCF-7 breast cancer cell growth. Oncogene 24: 6516-6524, 2005.

(i) $(9$ This work is licensed under a Creative Commons Attribution-NonCommercial-NoDerivatives 4.0 International (CC BY-NC-ND 4.0) License. 